\title{
New Dacron tissue colonisable keratoprosthesis: clinical experience
}

\author{
S Pintucci, F Pintucci, M Cecconi, S Caiazza
}

Department of

Ophthalmology,

S Giovanni Hospital,

Rome, Italy

S Pintucci

Department of

Ophthalmology,

Ophthalmic Hospital,

F Pintucci

M Cecconi

Department of

Ultrastructures,

Istituto Superiore di

Sanita', Rome, Italy

S Caiazza

Correspondence to:

Department of

Ultrastructures, Istituto

Superiore di Sanita', Viale

Regina Elena 299, 00161,

Rome, Italy. 24 April 1995
Rome, Italy

Dr Salvatore Caiazza,

Accepted for publication

\begin{abstract}
Background-Keratoprostheses (KPs) are made of an optical cylinder integrated with a supporting element which conditions their biocompatibility. A new KP with a Dacron tissue colonisable support has been designed in order to reduce significantly the complication rate. Methods-This new KP was implanted into 20 eyes of 20 patients with bilateral corneal blindness unsuited to a corneal implant. The follow up ranged from 24 to 96 months.
\end{abstract}

Results-All 20 patients had some improved visual acuity with 13 retaining this improvement for more than 2 years. Conclusion-These favourable results may be indicative of the reliability of the new KP.

(Br f Ophthalmol 1995; 79: 825-829)

A keratoprosthesis (KP) implantation is the last resort in severe corneal blindness not amenable to a corneal transplant. ${ }^{1}$ Although early visual recovery may be good, the percentage of severe complications with KPs is extremely high. ${ }^{12}$

KPs are generally made up of a polymethylmethacrylate (PMMA) optical cylinder, ${ }^{134}$ focusing the images on a functioning retina, integral with a supporting element fixing the $\mathrm{KP}$ to the eye.

The most important problem, which arises with the implantation of a $\mathrm{KP}$, is obtaining a perfect biological, mechanical, and functional

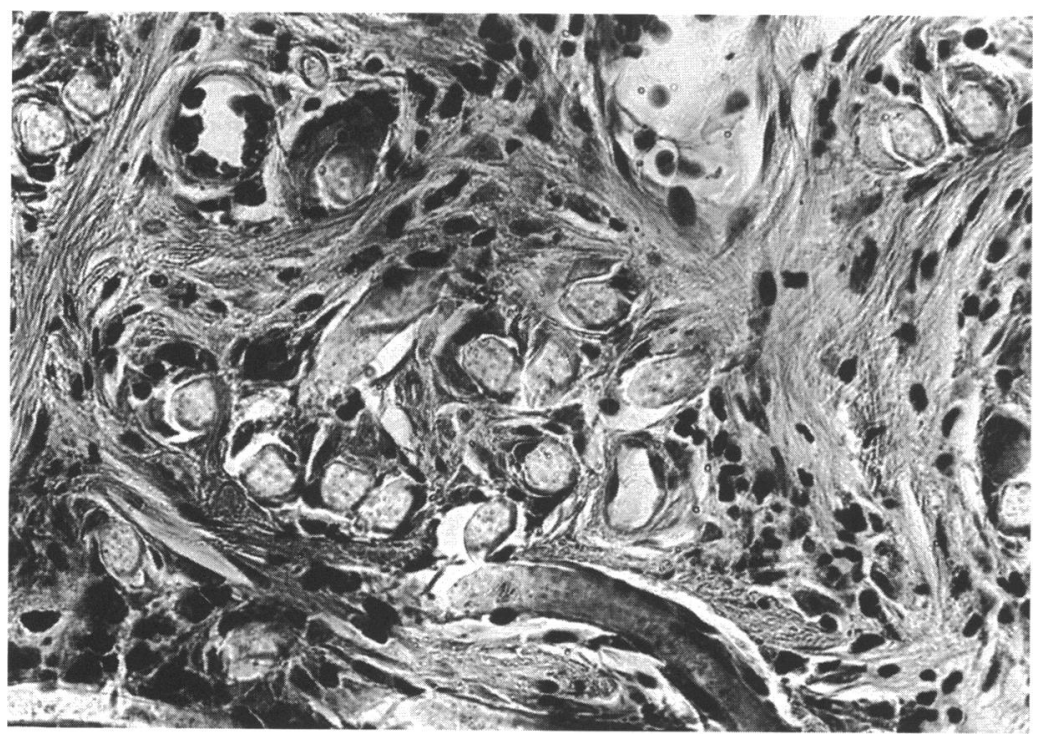

Figure 1 Cross section of Dacron filaments embedded in neoformed vascularised connective tissue before keratoprosthesis implantation. (LM, Masson's trichrome stain $\times 330$.) anchorage of the supporting element to eye tissues. However, the main complications are related to the eye's capability of extruding biomaterials only mechanically anchored but not biologically integrated, such as rigid $\mathrm{KP}$ supporting elements made of metal, plastic, ceramic, ${ }^{1-6}$ as well as hetero-, auto-, and homotissues (dentin, cartilage, bone) ${ }^{7-9}$ On the basis of previous studies, it can be stated that an ideal supporting element should fix the device without biological and mechanical adverse effects. ${ }^{81011}$

With the aim of avoiding the complications caused by interactions between the supporting element and the eye tissues, a new KP with a biointegratable supporting element ${ }^{12}$ was developed by Pintucci in $1979^{13}$ and the implantation technique is being constantly improved. ${ }^{14-16}$

In this new device, a Dacron supporting element ${ }^{12}$ is fixed, according to a personal technique (international patent pending), to a medical grade PMMA optical cylinder.

Previous experiments ${ }^{13}$ performed with human fibroblasts cultured in the presence of untreated Dacron tissue showed normal cells proliferating and adhering to the Dacron filaments. Dacron tissue implanted for 30 days under the conjunctiva of rabbit eyes, appeared to be colonised by a vascularised connective tissue filling any space within the Dacron weft. ${ }^{13-16}$ Moreover, specimens of Dacron tissue, placed under the lower lid skin for a period ranging from 20 to 40 days appeared to be tridimensionally colonised by autologous connective tissue (Figs 1 and 2).

On the basis of these results, we decided to implant our new KP in 20 eyes with bilateral corneal blindness not suitable for a corneal transplant.

\section{Materials and methods}

The KP is made of medical grade PMMA and of Sauvage filamentous Dacron fabric (nominal thickness $0.6 \mathrm{~mm}$, mean water porosity $1.600 \mathrm{ml}$ )

The weft of the Dacron tissue supporting element is fixed to a PMMA optical cylinder $5.4 \mathrm{~mm}$ long and $3.5 \mathrm{~mm}$ wide. The weft of the Dacron tissue is shown in Figure 3 and the assembled device and its nominal size are shown in Figures 4 and 5, respectively.

For inclusion in this study, bilateral corneal blindness not suitable for corneal transplant was required. All procedures were first time $\mathrm{KP}$ implants in a single eye with a severely vascularised cornea. All patients gave informed consent.

A preoperative diagnosis of ocular pemphigoid was made in 12 patients, three eyes had 


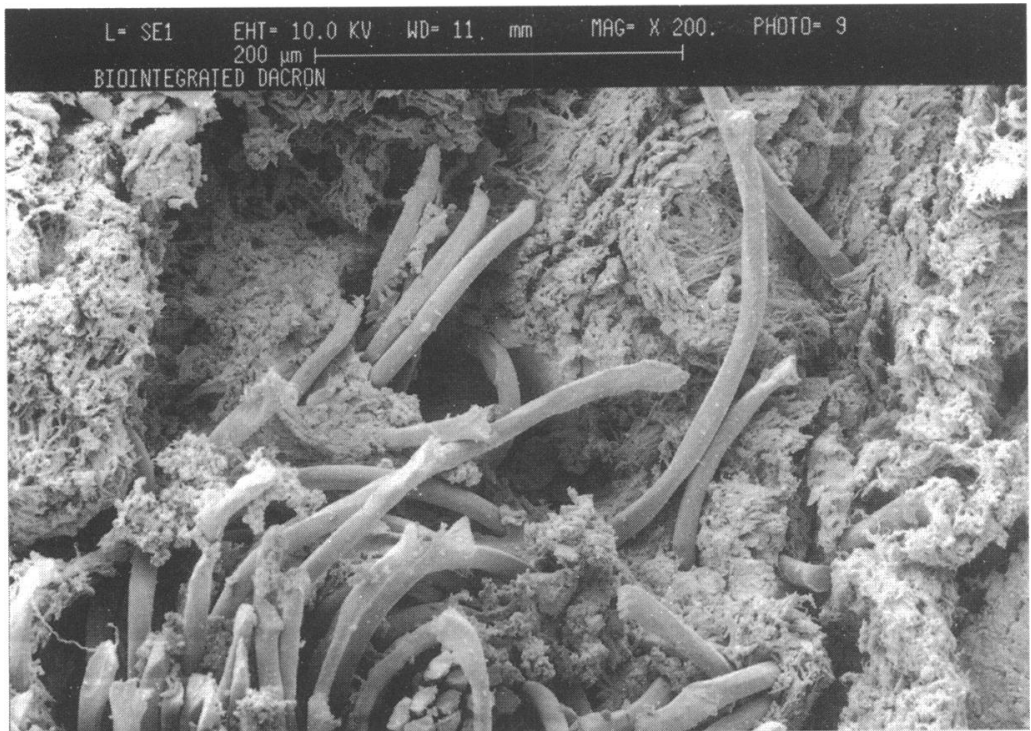

Figure 2 Dacron filaments embedded in neoformed vascularised connective tissue before keratoprosthesis implantation (scanning electron microscopy, $\times 165$ ).

trachoma, two eyes had recurrent severe herpes keratitis, and three eyes had alkali chemical burns. The diagnoses were made on the basis of clinical guidelines without relying on histopathological or immunological testing. Previous cataract surgery at the time of the initial KP operation was recorded in five patients.

Preoperative examination included vision testing, slit-lamp biomicroscopy, ultrasonography (A and B scans), evaluation of intraocular pressure by whichever means possible, electroretinogram and visual evoked potentials, Schirmer 1 test, and basic lacrimation test. Conjunctiva and lid examination is very important because reconstructive plastic surgery is often necessary before the KP implant, as in cases of trichiasis and lagophthalmos, etc.

The age of the patients at surgery ranged from 25 to 86 years with a mean of $57 \cdot 9$; there were 10 men and 10 women. All patients were in the chronic, non-inflammatory phase of the disease. All patients had cicatricial entropion with marked shortening (greater than $50 \%$ ) of

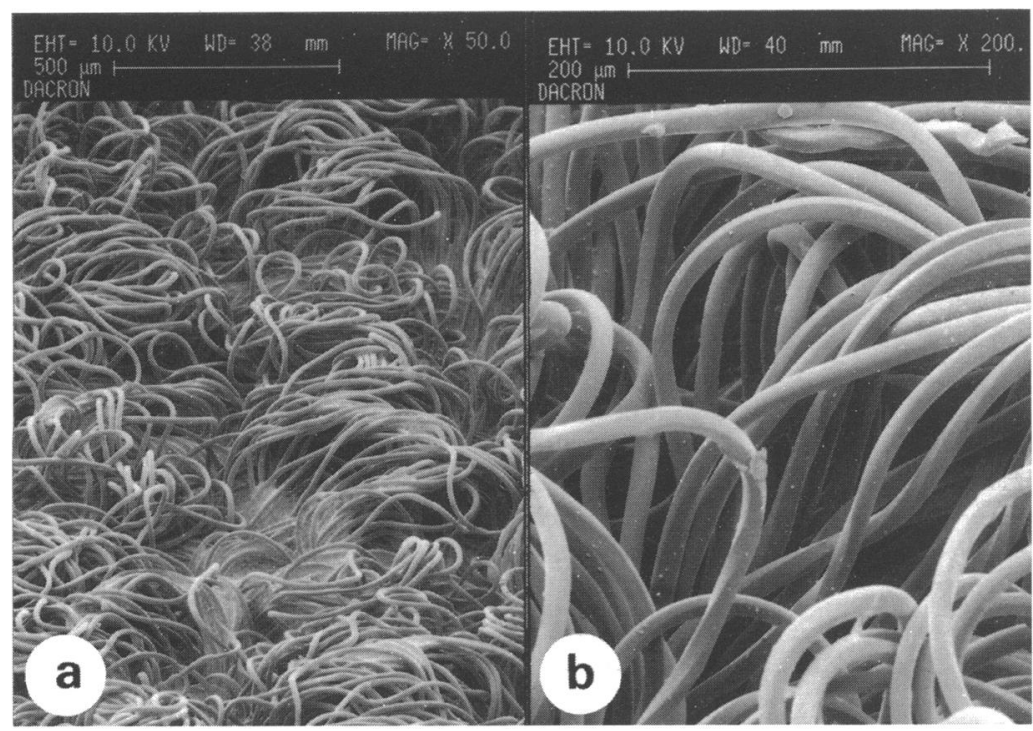

Figure 3 Weft (a) and enlargement of filaments of Sauvage filamentous Dacron fabric (b) (scanning electron microscopy, $\times 40$ and $\times 165$ ). conjunctival fornices, symblephara, and a variable amount of trichiasis. A history of glaucoma was obtained in one patient.

\section{TECHNIQUE}

The surgical technique of implanting a $\mathrm{KP}$ consists of two stages. In the first stage after eyedrop anaesthesia, the centre of the cornea is marked with gentian violet. General anaesthesia with nasal intubation is preferred.

In order to colonise the $\mathrm{KP}$, a $15 \mathrm{~mm}$ incision of the skin is performed in the inferior orbitopalpebral sulcus, the orbicular muscle fibres are spread apart to the orbitopalpebral septum and the $K P$ is introduced upside down with the optical cylinder vertical in the pocket. The orbicular muscle is sutured with 8.0 Dexon and the skin with 6.0 black silk.

If trichiasis, entropion, or symblepharon are present, palpebral and conjunctival plastic procedures must be performed. In dry eyes the lacrimal puncta are closed with diathermy. To expose the eye, two lid traction sutures are applied. The corneal epithelium is completely removed and a 10.0 Nylon suture is applied on the mark at the corneal centre for future reference.

A free buccal mucosal graft is dissected bearing in mind that once removed from the mouth, the mucosa shortens by one third in diameter. The buccal mucosa must also cover possible defects in the palpebral and bulbar conjunctiva.

Excessive submucosal fat is dissected away with fine scissors. The mucosa is washed and kept in a balanced salt solution-gentamicin solution. The oral mucosa is applied on the cornea whose epithelium is removed and sutured with Dexon 8.0. All blood clots are carefully removed. Antibiotic ointment is instilled and the lids closed. The dressing and a protective plastic shield are applied.

The second stage is performed after 2 months. Eye hypotony is obtained and general anaesthesia is performed. The colonised KP is removed from the lower lid. Under the operating microscope the excessive connective tissue that covers the optical cylinder is removed, the fixation of the optical part is tested, and the colonisation of the Dacron tissue is checked. The cornea is partly exposed dissecting the oral mucosal graft from the temporal-superior sector to the centre.

Once the cornea is exposed the KP optical cylinder site is stained with a $4 \mathrm{~mm}$ circular optical zone marker for corneal refractive surgery. With a diamond knife three partial thickness radial incisions are performed in the 2,6 , and 10 o'clock meridians. The cornea is trephined with Franceschetti trephine, then the radial incisions are completed. The iris is drawn down in the 6 o'clock direction and, with lateral movements, is extirpated, followed by cryoextraction of the lens.

The KP optical cylinder is positioned and the colonised Dacron tissue and the radial incisions are sutured. The oral mucosa is sutured to cover the KP and trephined to allow the passage of the anterior optical part. 


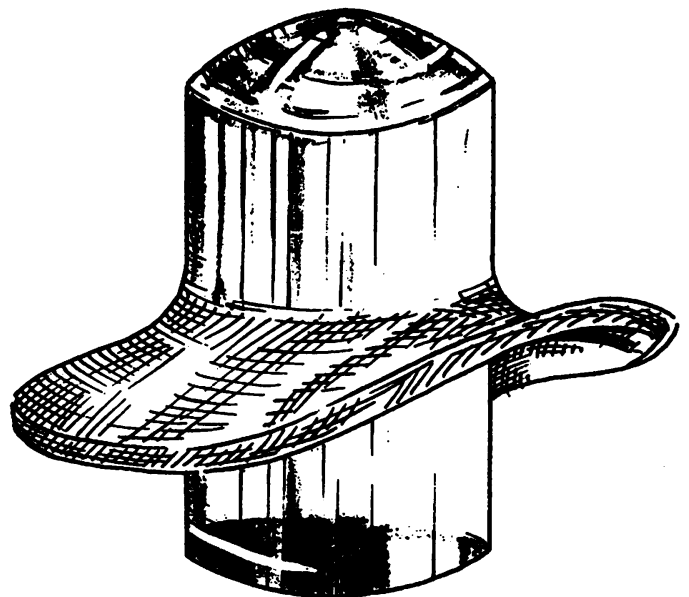

Figure 4 Assembled Pintucci's PMMA and Dacron keratoprosthesis.

In dry eyes, before the $\mathrm{KP}$ implantation in the eye, in order to protect the oral mucosa, the length of the palpebral fissure is reduced with a Blascovics lateral and a Streiff nasal tarsorrhaphy, leaving a central aperture for the optical cylinder.

Antibiotic ointment is instilled, and a protective plastic shield applied.

\section{Results}

We reviewed the charts of the 20 eyes of patients who underwent a Pintucci's transmucous KP implantation from January 1987 to December 1991. Follow up after surgery ranged from 24 to 96 months with a mean follow up of 58 months.

On preoperative examination the best corrected visual acuity was perception of light. A history of preoperative glaucoma was obtained in one patient.

In the follow up the appearance of the eye must be checked frequently. Follow up was done every week for the first 5 months after the $\mathrm{KP}$ was implanted, and thereafter once a month. Cases were reviewed with reference to the length of time the KP remained in situ, types and rates of complications, best postoperative aided visual acuity, and length of time vision was maintained.

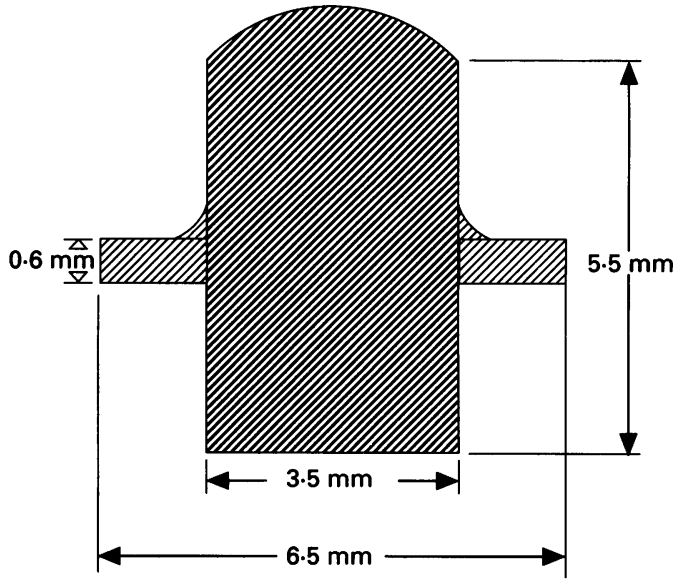

Figure 5 Nominal size of the Pintucci's keratoprosthesis.

In the follow up the position of the KP implant, tilt, and decentration were particularly observed. The Dacron tissue was also examined carefully for erosion through the surrounding tissues, loosening, aqueous leaks, and infection. Intraocular pressure was checked.

Postoperatively all the patients had an improved visual acuity and in particular 13 had a visual acuity of 0.4 or better. An improved visual acuity was maintained for more than 6 months in all the patients, for more than 1 year in 17, for more than 2 years in 15 , and improved vision is still present in 13 patients. The commonest complication was in 10 cases when an oral mucosal necrosis of varying degree with or without partial exposure had to be repaired surgically with a new oral mucosal graft in order to prevent extrusion. Nine required no further surgery after $\mathrm{KP}$ insertion. Other complications after KP insertion were one anterior epithelial overgrowth that occurred 38 months postoperatively, two retroimplant membranes or deposits that occurred after 52 and 25 months, one retinal detachment that occurred after 13 months, three choroidal detachments that occurred in the first month after $\mathrm{KP}$ insertion and that spontaneously regressed, one endophthalmitis 19 months after KP insertion, two KP spontaneous extrusions that occurred in two

Table 1 Complications

\begin{tabular}{|c|c|c|c|c|c|c|c|c|c|c|c|c|c|c|}
\hline No & $Y$ & Age & $S$ & $P P$ & $V A$ & $R$ & $F-U$ & $G N$ & $A M$ & $R M$ & $R D$ & $C D$ & $E N$ & $K E$ \\
\hline 1 & 85 & 55 & $\mathbf{F}$ & $\mathbf{P}$ & 0.5 & +4 & 62 & + & - & - & - & - & - & + \\
\hline 2 & 87 & 25 & $\mathbf{M}$ & $\mathrm{P}$ & 0.5 & -5 & 75 & - & - & + & - & - & - & - \\
\hline 3 & 87 & 51 & $\mathbf{M}$ & $P$ & 0.7 & -10 & 70 & - & - & - & - & - & - & - \\
\hline 4 & 87 & 74 & $\mathrm{~F}$ & $\mathbf{P}$ & 0.1 & +3 & 62 & - & - & - & - & - & - & - \\
\hline 5 & 88 & 55 & $\mathbf{M}$ & $T$ & 0.3 & -8 & 34 & + & - & - & - & - & - & + \\
\hline 6 & 88 & 50 & $\mathbf{M}$ & HK & 0.3 & -6 & 40 & + & - & + & - & + & - & - \\
\hline 7 & 89 & 59 & $\mathbf{F}$ & $\mathrm{AB}$ & $0 \cdot 1$ & +20 & 47 & + & - & - & - & - & - & - \\
\hline 8 & 89 & 74 & $\mathbf{F}$ & $\mathbf{P}$ & 0.04 & 0 & 46 & - & - & - & - & - & - & - \\
\hline 9 & 89 & 76 & $\mathbf{F}$ & $\mathbf{P}$ & $0 \cdot 1$ & -4 & 42 & + & - & - & - & - & - & - \\
\hline 10 & 89 & 63 & $\mathbf{M}$ & $\mathbf{P}$ & 0.4 & 0 & 34 & + & - & - & + & - & + & - \\
\hline 11 & 89 & 59 & $\mathbf{M}$ & AB & 0.7 & -13 & 50 & - & + & - & - & + & - & - \\
\hline 12 & 89 & 79 & $F$ & $\mathbf{P}$ & 0.4 & -11 & 49 & - & - & - & - & - & - & - \\
\hline 13 & 89 & 33 & $\mathbf{M}$ & $\mathbf{P}$ & 0.5 & -2 & 49 & + & - & - & - & + & - & - \\
\hline 14 & 89 & 33 & $\mathbf{F}$ & $\mathbf{P}$ & LP & 0 & 47 & - & - & - & - & - & - & - \\
\hline 15 & 90 & 57 & $\mathbf{M}$ & $\mathrm{T}$ & $\overline{0.6}$ & -2 & 34 & + & - & - & - & - & - & - \\
\hline 16 & 90 & 67 & $\mathbf{M}$ & $\mathbf{A B}$ & 0.5 & -3 & 38 & + & - & - & - & - & - & - \\
\hline 17 & 90 & 37 & $\mathrm{~F}$ & $\mathbf{P}$ & $0 \cdot 2$ & -10 & 34 & - & - & - & - & - & - & - \\
\hline 18 & 91 & 86 & $\mathbf{F}$ & $P$ & $0 \cdot 2$ & -9 & 39 & + & - & - & - & - & - & - \\
\hline 19 & 91 & 60 & $\mathrm{~F}$ & HK & 0.4 & -6 & 36 & - & - & - & - & - & - & - \\
\hline 20 & 91 & 65 & $\mathbf{M}$ & $\mathrm{T}$ & 0.1 & -2 & 34 & - & - & - & - & - & - & - \\
\hline
\end{tabular}

$Y=$ year of implantation; Age=age when implanted; $S=$ sex; $P P=$ primary pathology: $P=$ pemphigoid, $T=$ trachoma, $H K=$ herpes keratitis, $A B=$ alkali burn; $V A=$ postoperative visual acuity; $R=$ postoperative correction; $F-U=$ months of follow up; $G N=$ oral mucosal graft necrosis; $\mathrm{AM}=$ anterior membrane; $\mathrm{RM}=$ retroprosthetic membrane; $\mathrm{RD}=$ retinal detachment; $\mathrm{CD}=$ choroidal detachment; $\mathrm{EN}=$ endophthalmitis; $\mathrm{KE}=$ keratoprosthesis extrusion. 
patients who were lost to follow up and who refused to have a new oral mucosal graft because of good visual acuity (see Table 1).

\section{Discussion}

A keratoprosthesis implantation is the last resort to regain useful visual acuity in severe corneal blindness caused by trauma, chemical burns, infections, trachoma, ocular pemphigoid, Stevens-Johnson syndrome as well as severe dry eyes, repeated graft failures, etc, and when a corneal transplant cannot be attempted or has repeatedly failed.

None of the patients had a bullous keratopathy, which we consider a poor indication for a $\mathrm{KP}$ implantation because it is amenable to keratoplasty.

KPs basically consist of an optical cylinder, penetrating the cornea and focusing the images on the retina, integral with a supporting element fixing the device to the eye.

Although early visual recovery may be good severe complications, such as extrusion, are extremely high in spite of the number of devices and surgical techniques described in literature.

Some problems with the long term performance of KPs have not yet been solved and, unfortunately, worldwide research in this field is very limited. 12

Since 1789, several KPs have been developed and from the clinical results of a very large number of different implants it can be recognised that PMMA has been the most widely used material for optical cylinders and it has not been reported as a cause of failure. ${ }^{134}$ On the contrary, most complications are due to rigid supporting elements which are simply sutured and/or screwed to the cornea. In these devices the mechanical stress leads to inflammation with tissue melting and to the formation of empty spaces at the implant-eye interface. As a consequence, aqueous humour leakage, infections, and reparative epithelial and connective tissue proliferation may occur, with subsequent encapsulation, formation of retroprosthetic membranes, and KP extrusion. 481117

Many biological materials, autologous (fascia lata, periosteum, conjunctiva, Tenon's capsule, cornea, labial mucus, cartilage, eyelid skin) and homologous (cornea and sclera) have been used for covering rigid supporting flanges ${ }^{1468}$; however, in 1979 we developed a new KP with a Dacron tissue supporting element with the aim of reducing significantly the complication rate. Since 1954, Dacron fabrics have been used successfully in permanent cardiovascular prostheses which were colonised by host tissues. ${ }^{12}$

Previous in vitro experiments performed with human lung fibroblasts cultured in the presence of untreated Dacron tissue showed normal cells proliferating and adhering to the Dacron filaments. The same tissue implanted for 30 days under the conjunctiva of rabbit eyes, appeared to be colonised by a vascularised connective filling any space within the Dacron weft. ${ }^{13-16}$ Moreover, specimens of Dacron tissue, placed under the lower lid skin for a period ranging from 20 to 40 days before implantation on the eye and observed with light microscopy and scanning electron microscopy, appeared to be tridimensionally colonised by autologous connective tissue (Figs 1, 2).

After extensive in vitro and in vivo testing of different kinds of Dacron tissues, the Sauvage filamentous Dacron fabric was chosen for its good colonisability and mechanical performance. The main characteristics of this Dacron tissue are: it is soft and pliable (thus preventing aseptic corneal necrosis by mechanical stress); chemically inert; not subject to resorption; does not activate the complement (to some extent); can be autoclaved; can be easily cut in the desired shape; and can be sutured. ${ }^{12}$

Human fibroblasts in vitro proliferate, adhering to the Dacron filaments without cellular damage. ${ }^{13}$ In vivo the Dacron tissue is colonised by a vascularised connective tissue migrating from the surrounding tissues, filling completely the free spaces among the filaments.

It must be stressed that reparative epithelial proliferation along the optical cylinder is stopped owing to contact inhibition ${ }^{17}$ and to lack of empty spaces which have been filled by the neoformed connective tissue.

Results were gauged by measuring the visual acuity, the length of time improved vision is retained, and the time the KP remains in situ. Visual acuity itself is not necessarily a criterion of success or failure, as good guiding vision may be achieved without central vision. Complications must be overcome by careful follow up and prompt action.

It is difficult to predict which cases will be successful. Patients thought to have a very poor prognosis may do well. In general patients with good tear secretion do better than patients with dry eyes.

Contrary to what has been suggested by some authors, ${ }^{3} 101819$ we do not graft the supporting flange in the corneal stroma thickness but we suture it on the corneal surface. In this way the colonised Dacron tissue plays a trophic and a mechanical role. It is important to underline that extremely thin and vascularised corneas may be treated successfully in this way too.

The oral mucosa employed to cover the colonised Dacron flange shows best results probably because it is more vascularised and has a faster cellular turnover than skin, conjunctiva, ${ }^{20}$ and other tissues.

It must be stressed that the colonised Dacron tissue KP implant can be considered and behaves as an autotransplant placed between the cornea and the oral mucosal graft. It heals, becoming biologically and mechanically fully integrated with the surrounding tissues, and also acts as a barrier to microbial contamination. It is, in fact, well known that biomaterials, as well as traumatised tissues, may be a pabulum for bacteria leading to infections typically associated with prosthetic devices. ${ }^{21} 22$

Infections occur in all kinds of implanted devices and are related to the biomaterials 
which lower the natural defences of the organism. In addition they promote a preferential adhesive colonisation ${ }^{21}$ complicated by enhanced antibiotic resistance owing to the modifications of the saprophytic behaviour of bacteria. ${ }^{21-24}$ Inflammation, aseptic necrosis, lack of mechanical and biological integration, filtration of anterior chamber aqueous humour predispose to implant failure.

We think that this new device may be considered as a real step on the way to overcoming the apparently inseparable difficulties represented by KP mechanical anchorage and biointegrability.

1 Polack FM. Corneal optical prostheses. Br $f$ Ophthalmol 1971; 55: 838-43.

2 Roper-Hall MJ. Why are keratoprostheses not given more importance? Eur f Ref Surg 1991; 3: 79.

3 Stone W, Herbert E. Experimental study of plastic material as replacement for cornea. Preliminary report. $A m \mathcal{F}$ Ophthalmol 1953; 36: 68-73.

4 Fyodorov SN, Moroz ZI, Zuev VK. Keratoprostheses. London: Churchill Livingstone, 1987: 7-54.

5 Cardona H. Keratoprosthesis with a plastic fiber meshwork supporting plate. Am $\mathcal{F}$ Ophthalmol 1967; 64: 228-33.

6 Polack FM. Clinical results with ceramic keratoprosthesis. Cornea 1983; 2: 185-96.

7 Strampelli B. Keratoprosthesis with osteodontal tissue. $\mathrm{Am}$ f Ophthalmol 1963; 89: 1029-39.

8 Strampelli B. Nouvelle orientation biologique dans la keratoprosthese. Bull Mem Soc Fr Ophtalmol 1964; 77: 145-61.

9 Hatt M. Experimental osteokeratoprosthesis. Ophthalmol Res 1979; 11: 40-51.
10 Knowles W. Effect of intralamellar plastic membranes on corneal physiology. Am f Ophthalmol 1961; 51: 274-84.

11 Turss R, Friend J, Dohlman CH. Effect of corneal fluid barrier on nutrition of the epithelium. Exp Eye Res 1970; 9: 254 .

12 De Bakey ME, Crawford ES, Morris GC, Cooley DA. Patch graft angioplasty in vascular surgery. $f$ Cardiovasc Surg 1962; 3: 106-41.

13 Caiazza S, Fanizza C, Mazziotti I, Pintucci S. Light and scanning electron microscopy evaluation of the Dacron felt as the haptic part of an improved keratoprosthesis. An felt as the haptic part of an improved keratoprosthesis.

14 Pintucci S, Pintucci F, Caiazza S. The Pintucci's biointegrable keratoprosthesis: an up to date. Bologna: Studio ER Congressi, 1990: 137-42.

15 Pintucci S, Caiazza S, Donelli G. Biointegrable keratoprostheses: performances and recent improvements. Ital $\mathcal{f}$ Ophthalmol 1993; 7: 13-20.

16 Pintucci S, Pintucci S, Caiazza S. The Dacron felt colonizable keratoprosthesis. Refract Corneal Surg 1993; 9: 196-7.

17 Alberts B, Bray D, Lewis J, Raff M, Roberts K, Watson JD. Cell growth and division. In: Molecular biology of the cell. 2nd ed. New York: Garland, 1983: 618-9.

18 Stone W. Study of patency of openings in corneas anterior to intralamellar plastic artificial discs. Am $₹$ Ophthalmol to intralamellar plast

19 Cardona H. Keratoprosthesis: acrylic optical cylinder with supporting intralamellar plate. Am $\mathcal{F}$ Ophthalmol 1962; 54: 284-94.

20 Kozarsky AM, Knight SH, Waring GO. Clinical results with a ceramic keratoprosthesis placed through the eyelid. Ophthalmology 1987; 94: 904-11.

21 Gristina AG. Biomaterial-centered infection: microbial adhesion versus tissue integration. Science 1987; 237: adhesion

22 Oga M, Sugioka Y, Hobgood CD, Gristina AG, Myrvik QN. Surgical biomaterials and differential colonization by Staphylococcus epidermidis. Biomaterials 1988; 9: 285-9.

23 Lowy FD, Hammer SM. Staphylococcus epidermidis infections. Ann Intern Med 1983; 99: 834-9.

24 Moller JK. Drug resistance and plasmid profiles in Staphylococcus epidermidis in 1964 and 1986. F Hosp Infect 1988; 12: 19-27. 